\title{
Causes of academic and behavioral difficulties among Japanese-Brazilian students: cognitive, linguistic and parental education factors
}

\author{
Yukiko Konda ${ }^{*}$, Yui Miura ${ }^{2}$ and Manabu $\mathrm{Oi}^{3}$
}

\author{
* Correspondence: \\ u555069e@alumni.osaka-u.ac.jp \\ ${ }^{1}$ United Graduate School of Child \\ Development, Osaka University, \\ Kanazawa University, Hamamatsu \\ University School of Medicine, \\ Chiba University and University of \\ Fukui, Yamadaoka Suita, Osaka \\ 565-0871, Japan \\ Full list of author information is \\ available at the end of the article
}

\begin{abstract}
As in other countries with large immigrant populations, in Japan there is increasing interest in students from culturally and linguistically diverse backgrounds who experience learning and behavioral difficulties in the classroom. The causes of the problems have not been identified owing to a lack of appropriate and comparable assessments. In this study we focused on Japanese-Brazilian elementary school students, the largest ethnic minority group of students in Japan, and explored whether evaluation of their learning and behavior is influenced by the following aspects: language proficiency, cognitive capacity and family environment. We conducted a set of tasks to assess the students' vocabulary and syntactic development in Japanese and Portuguese, and to evaluate their executive functions as an indicator of nonverbal cognitive development. Questionnaires were administered to teachers and parents in order to gain information about the children's classroom performance and family environment, such as parents' educational background and place of birth. Three key findings from our data include the following. (1) Some students in mainstream classrooms were 'at-risk' in their level of cognitive capacity. (2) Even among those with typical cognitive capacity, academic performance and classroom behavior were still rated lower than at grade level for the native population. (3) Teachers' ratings of the two aspects were associated with different factors: low academic performance was significantly influenced by poor working memory, while problematic behavior was affected by Japanese grammatical skill and father's educational background. This study used quantitative data to confirm that clear information about students' linguistic, cognitive and environmental profiles, could be used to ensure that appropriate support is provided to students experiencing difficulties.
\end{abstract}

Keywords: Culturally and linguistically diverse students; Academic performance; Behavioral problems; Cognition; Language; Parental education; Executive functions; Working memory; Japanese-Brazilian

\section{Background}

Since the amendment of the Immigration Control and Refugee Recognition Act in Japan in 1990, the number of foreign residents, including those of Japanese ancestry accompanied by students, has been steadily increasing. There are currently 71,545 foreign students enrolled in public schools in Japan. Of these, 27,013 are reported to require Japanese language instruction, with the largest group (32.8\%) being those whose mother tongue is Portuguese, the official language spoken in Brazil (Ministry of

\section{Springer}

(c) 2015 Konda et al.; licensee Springer. This is an Open Access article distributed under the terms of the Creative Commons

Attribution License (http://creativecommons.org/licenses/by/4.0), which permits unrestricted use, distribution, and reproduction in any medium, provided the original work is properly credited. 
Education, Culture, Sports, Science and Technology-Japan [MEXT] 2012a, b). The term 'foreign resident' is generally favored in Japan over the word immigrant, because the government has no clear and consistent policy to deal with people moving across national borders (Kitawaki 2008). In accordance with previous studies (Cummins 2009; Sullivan 2011), this article uses the term 'culturally and linguistically diverse (CLD)' students to refer to foreign child residents with diverse cultural and linguistic backgrounds.

As reported in other countries, many CLD students have learning and behavioral difficulties (Ceballos and Bratton 2010; Dunn 1968; Pierce et al. 2013; Sullivan 2011). Researchers have argued that the problems faced by these students are complex, making assessment of underlying causes a challenge. In Japan, the situation involves considerable trial and error, and sufficient quantitative research data are lacking. The following section reviews the situation regarding CLD students and difficulties with their assessment.

\section{Issues regarding the assessment of learning and behavioral difficulties}

Previous studies have discussed factors causing difficulties in learning and behavior among CLD students, pointing out that their language proficiency and cultural/social background may have considerable impact on their classroom performance (Georgiades et al. 2007; Klingner and Edwards 2006). At the same time, there is growing awareness of the possibility that a certain proportion of children may have developmental disorders (Norbury and Alison 2013). Cognitive assessment batteries are generally used for identifying developmental delay, but performance is strongly influenced by the language used for instructions and cultural setting during administration (Gunderson and Siegel 2001; Norbury and Alison 2013). There are currently few appropriate methods to assess the development of CLD children, making it a challenge to determine which children need to be referred to specialists.

As the need for early intervention has become more widely acknowledged, there has been an attempt in the United States to evaluate children not just by referring to the results of standardized (e.g., IQ) tests, but rather by observing children's overall classroom skills, using a model called 'Response to Intervention' (RTI; Hughes and Dexter 2011). This model focuses on both academic learning and behavioral performance of monolingual and CLD students (Carter-Smith 2008), and encourages cooperative assessment and support by school staff (Bradley et al. 2007). While the RTI model can effectively assess and support students challenged in the classroom, several limitations have been pointed out. Reynolds and Shaywitz (2009) noted that it lacks firm evidence. Harry and Klingner (2014) reported that CLD students are more frequently diagnosed as having learning disabilities and are overrepresented in special education. Conversely, Hibel and Jasper (2012) are concerned about CLD students placed in mainstream classes, when they may benefit from being in a classroom for children with special educational needs, and have already been disadvantaged owing to delayed intervention.

Researchers such as Sullivan (2011) have mentioned that more than 40 years after being identified by Dunn (1968), problems of assessment and support still cause a disproportionate representation of CLD students in special education. The system used to evaluate CLD students in Japan lags behind that of western countries. Students with poor Japanese language ability tend to be suspected of having developmental disorders 
when they are not able to follow lessons in Japanese. On the other hand, there are cases where even though the symptoms of developmental disorders are present, the problem is thought to be caused solely by a low level of Japanese proficiency (Nii and Makoto 2013). Abe (2007) argued that inappropriate intervention may mean that children's educational outcomes are even more negatively affected. Research conducted in Japan to date has been limited mainly to surveys focusing on language proficiency, and reports based on individual case studies. There is an urgent need to collect objective data that can be used to develop assessment methods to identify the factors underpinning individual problems in order to prevent inappropriate or delayed educational placement due to over- or underdiagnosis.

The inadequate grasp of factors contributing to children's difficulties is partly due to the lack of appropriate indicators of cognitive development. Recent research has attempted to incorporate indicators of nonverbal cognitive capacity and verbal working memory in CLD and monolingual children (Engel de Abreu et al. 2013; Pina et al. 2014). Pina et al. (2014) explored how children's mathematical performance is affected by working memory and verbal language ability, general intelligence, and socioeconomic status. Their results revealed an association between verbal working memory and arithmetic ability, and also between parental educational level and the grasp of quantitative concepts. Using cognitive data has enabled these studies to explain the association between students' language acquisition and environment.

The present study aimed to examine the causes of learning and behavioral difficulties among Japanese-Brazilian students who speak Portuguese as their first language. Difficulties were investigated from three main perspectives: cognitive development, language acquisition and home environment, none of which can be observed purely through classroom behavior.

\section{Cognitive development perspective}

The cognitive abilities of information processing, memory and problem solving are indispensable functions when learning. Cognitive delays may suggest the presence of developmental disorder. Early identification and support appropriate to the nature of the difficulties are necessary. However, as many current psychological batteries are strongly influenced by language and culture, they may not be appropriate for accurate assessment of CLD students' cognitive abilities. A key challenge is how to assess the cognitive development of children beyond what can be understood from the classroom environment.

Recent work has considered the effect of the brain's executive function on academic achievement and behavioral problems (Miyake and Friedman 2012; St Clair-Thompson et al. 2006; Vuontela et al. 2013). According to Lezak (1982, p.281), executive function refers to "mental capacities necessary for formulating goals, planning how to achieve them, and carrying out the plans effectively". Individuals with developmental disorders also have problems with executive functions (Corbett et al. 2009; Pennington and Ozonoff 1996), and executive function as a whole predicts IQ (Brydges et al. 2012), although not all executive tasks are related to IQ (Friedman et al. 2006). Furthermore, Gathercole et al. (2008) suggest that working memory, part of executive function, plays a more significant role in typical classroom activities than IQ. 
Luciana and Nelson (2002) conducted visual memory and executive function tasks using the Cambridge Neuropsychological Test Automated Battery (CANTAB) and other nonverbal cognitive tasks. English as a second language (ESL) students aged 6-12 years were tested and compared with monolingual English-speaking students. The results did not show any between-group differences, apart from differences in Vocabulary scaled scores on the Wechsler Intelligence Scale for Children - Third Edition (WISC-III). They reported correlations between performance on subtests of CANTAB and WISC-III Block Design scaled scores. CANTAB scores were shown to be useful in identifying children with developmental disorders such as attention deficit hyperactivity disorder (ADHD) and autism spectrum disorder (Luciana 2003). A study using CANTAB was also conducted with Brazilian children. Roque et al. (2011) presented students aged 6-11 years with visual memory and executive function tasks. They reported an association between cognitive development and the children's chronological age. These studies suggest that it may be possible to carry out cognitive assessment using CANTAB with JapaneseBrazilian students with learning and behavioral difficulties. CANTAB tasks may be less affected by linguistic and cultural factors, and may be more appropriate to assess CLD children acquiring a second language. Where results indicate cognitive ability within the typical range, it would be appropriate to look beyond developmental disorders and consider language skills and home environment. If results show that students' cognitive capacity is within the 'at-risk' range, they would need support.

\section{Language acquisition perspective}

It is well known that while day-to-day conversation of CLD students develops relatively smoothly, it typically takes more than 5 years for academic language proficiency to develop to peer level (Cummins 1981, 2014). From a different perspective, research into bilingual fluency has shown that balanced bilingual students have advantages over monolingual students in academic performance (Golash-Boza 2005).

Before CLD students develop sufficient academic language proficiency, they face difficulties in the classroom. A survey conducted in the United States revealed that 29\% of eighth grade ESL students reached average academic achievement standards (Kohler and Lazarín 2007). Other studies have found that students' behavioral problems are influenced by language ability (Petersen et al. 2013). Problems in intercultural communication exacerbated by limited language proficiency, e.g., not understanding the teacher's instructions or friends or not being able to express feelings, may lead to the behavioral difficulties noted in the literature.

Cummins (1981) emphasized the importance of gaining information about CLD students' level of classroom language and their mother tongue, suggesting that this knowledge is important for setting up learning activities. Where children are skilled in their first language, implementing some activities using this relative strength can mitigate learning difficulties. However, it is likely that in some cases, proficiency in both first and second languages will be limited. In this case, the presence of a language disorder should be considered, and if such a diagnosis is confirmed, then intervention should be implemented. When considering the cause of learning and behavioral problems in CLD children, teachers and specialists should assess abilities in both languages. 


\section{Home environment perspective}

One indicator that predicts CLD students' learning and behavioral difficulties is the home environment. It is known that the socioeconomic status of immigrant families has a great impact on children's difficulties (Duncan et al. 1994; Bradley and Corwyn 2002). CLD students in low-income families are reported to exhibit learning and behavioral problems more frequently (Ceballos and Bratton 2010). Parents' educational background and involvement in child rearing also have an impact on the children's academic achievement and behavioral problems (Bradley et al. 2001; Suárez-Orozco et al. 2009; Villiger et al. 2014).

Much research regarding parental factors has focused on mothers. For example, Hammer et al. (2012) reported that mothers' characteristics have an effect on children's linguistic development. Suárez-Orozco et al. (2009) stated that mothers' educational background predicts children's academic achievement. Similarly, a mother's educational background has an impact on her child's behavior (Edwards 2014). In contrast, some research indicates the importance of paternal factors on children's classroom performance. McFadden et al. (2011) stated that the involvement of low-income fathers with their children's education predicts their academic achievement.

As much of this work has been carried out in western countries, it would be valuable to now examine the Japanese context. This should involve consideration of both the influence of home environment and parents' educational background on students' learning and behavior, including gender differences.

\section{Aims and research questions}

This study explored factors that could influence learning and behavioral difficulties experienced by Japanese-Brazilian students. The aim was to first collect quantitative data regarding nonverbal cognitive capacity, language proficiency (in both the first and second language) and parental background. We then examined the relationship between those factors and children's academic and behavioral performance, using statistical methods. The following research questions were posed:

1. Are there Japanese-Brazilian students who are enrolled in mainstream classes, but at risk of cognitive dysfunction?

2. Are there Japanese-Brazilian students evaluated as having low academic achievement and behavioral difficulties in the absence of a recognized cognitive disorder?

3. Are limited academic progress and behavioral difficulties influenced by three predictors: cognition, language and parental education?

\section{Results}

Students with risk of cognitive dysfunction

Of the 81 Japanese-Brazilian students recruited, seven students had previously been diagnosed with a disability (five with intellectual disability and one each with ADHD and unspecified developmental delay). For the remaining children placed in mainstream classes without any diagnosis of developmental delay, we considered whether any were at risk of cognitive delay. Six students $(8 \%)$ were identified as being in the clinical/borderline range for suspected cognitive disorders. 


\section{Academic and behavioral performance in children with/without risk}

Based on the presence or absence of diagnosis and scores obtained from the CANTAB tests, we classified participants into three groups: Typical, At-risk and Diagnostic Groups. Table 1 shows the results of the teachers' ratings for each group and results of nonparametric analysis, comparing the scores among the groups.

Chronological ages were not statistically different across groups. Group differences in the teachers' ratings for both academic and behavioral performance across groups, were noted. The academic performance of students in the At-risk Group was significantly lower than that of the Typical Group $(U=297, p=0.03$; the p-value showed the results of one-tailed tests). With regard to behavioral problems, the Typical Group score significantly lower than the At-risk group $(U=118, p=0.04)$, while the score of the At-risk Group was also significantly lower than the Diagnostic Group ( $U=32.5, p=0.05)$. Results suggest that children who have been diagnosed or are at risk of cognitive disorders exhibit more problematic behavior in the classroom than children with no cognitive delay.

Table 2 shows the number and percentage of children whose academic performance is below grade level, and children whose behavioral difficulties fall into the clinical/borderline range. For academic performance, an average grade level is 6 . Results revealed that more than $70 \%(N=59)$ of CLD children (70.6\% even in the Typical Group) scored lower than the average level. For behavioral performance, the cutoff point of the T-score (60) was used to screen children with a considerable level of behavioral difficulties. It was found that more than $35 \%(N=29)$ of the total sample (and $29.4 \%$ of the Typical Group) have behavioral problems.

\section{Influence of cognitive, linguistic and parental factors}

Children in the Typical group did not show any cognitive abnormalities but still received poorer ratings from teachers. We aimed to explore factors that influence these ratings of classroom performance. Table 3 shows descriptive statistics of children in the Typical Group, including scores obtained from cognitive and language tasks and parental education data. The distribution of gender and birthplace was also considered. In the Typical Group $51.5 \%$ of the children were male $(N=35)$ and $49.5 \%$ were female $(N=33)$. For

Table 1 Teachers' ratings of participants and Mann-Whitney analysis $(\mathrm{N}=\mathbf{8 1})$

\begin{tabular}{|c|c|c|c|c|c|c|c|c|c|c|}
\hline & \multicolumn{3}{|c|}{$\begin{array}{l}\text { A: Typical } \\
(n=68)\end{array}$} & \multicolumn{3}{|c|}{$\begin{array}{l}\text { B: At risk } \\
(n=6)\end{array}$} & \multicolumn{3}{|c|}{$\begin{array}{l}\text { C: Diagnostic group } \\
(n=7)\end{array}$} & \multirow[t]{2}{*}{$\begin{array}{l}\text { Mann- } \\
\text { Whitney }^{c}\end{array}$} \\
\hline & $\bar{M}$ & Range & $\overline{S D}$ & $\bar{M}$ & Range & $\overline{S D}$ & $\bar{M}$ & Range & SD & \\
\hline Age (years) & 9.43 & $6.2-11.9$ & 1.65 & 8.98 & $6.2-11.5$ & 2.07 & 8.86 & $7.3-10.5$ & 1.24 & $A=B=C$ \\
\hline \multirow{3}{*}{$\begin{array}{l}\text { Academic } \\
\text { performance }^{a}\end{array}$} & \multirow[t]{3}{*}{4.62} & \multirow[t]{3}{*}{$2-10$} & \multirow[t]{3}{*}{1.64} & \multirow[t]{3}{*}{3.33} & \multirow[t]{3}{*}{$2-5$} & \multirow[t]{3}{*}{1.21} & \multirow[t]{3}{*}{4.43} & \multirow[t]{3}{*}{$2-8$} & \multirow[t]{3}{*}{2.23} & $A>B^{d}$ \\
\hline & & & & & & & & & & $A=C$ \\
\hline & & & & & & & & & & $B=C$ \\
\hline $\begin{array}{l}\text { Behavioral } \\
\text { problems }\end{array}$ & 55.83 & $41-76$ & 8.61 & 60.83 & $53-67$ & 5.15 & 66.86 & $58-80$ & 7.36 & $A<B<C^{d}$ \\
\hline
\end{tabular}

${ }^{a}$ The teachers rated the current academic performance of students using a five-point scale: 1) far below grade level; 2 ) somewhat below grade level; 3) at grade level; 4) somewhat above grade level; and 5) far above grade level. The subjects rated were mathematics and Japanese language (academic performance range 2-10). ${ }^{\mathrm{b}}$ The clinical/borderline level of behavioral problems was set as TRF T-score $\geq 60$ points.

c.The Wilcoxon rank-sum (Mann-Whitney) test was conducted.

${ }^{\mathrm{d}}$ The $p$-values $(<0.05)$ shown are the results of one-tailed tests. 
Table 2 Academic performance below grade level and Behavioral Clinical/borderline level $(\mathbf{N}=\mathbf{8 1})$

\begin{tabular}{|c|c|c|c|c|c|c|}
\hline & \multicolumn{2}{|c|}{ Typical $(n=68)$} & \multicolumn{2}{|c|}{ At risk $(n=6)$} & \multicolumn{2}{|c|}{ Diagnostic group $(n=7)$} \\
\hline & $\mathbf{N}$ & $\%$ & $\mathbf{N}$ & $\%$ & $\mathbf{N}$ & $\%$ \\
\hline Academic performance below grade level & 48 & 70.6 & 6 & 100.0 & 5 & 71.4 \\
\hline Behavioral Clinical/borderline level & 20 & 29.4 & 3 & 50.0 & 6 & 85.7 \\
\hline
\end{tabular}

birthplace, $61.5 \%$ of the students $(N=40)$ were born in Japan. Using all the data as possible explanatory variables, we performed multiple regression analysis to explore the predictors of academic and behavioral rating. A total of 68 participants in the Typical Group were included in the analysis. The stepwise backward multiple regression method was adopted.

Table 4 summarizes the explanatory variables that significantly affected academic performance and behavioral difficulties. Scores in the digit span task in Japanese were significantly associated with academic performance. The estimates showed that scores for academic performance increased by 0.181 points with an increase of one point in digit span in Japanese. For the behavioral problems, paternal education and scores on the

Table 3 Cognitive and language proficiency and environment of participants of Typical Group ( $\mathbf{N}=68$ )

\begin{tabular}{|c|c|c|c|}
\hline Variable & $M$ & Range & SD \\
\hline Age (years) & 9.43 & $6.2-11.9$ & 1.65 \\
\hline \multicolumn{4}{|l|}{ Cognitive capacity $^{a}$} \\
\hline Spatial Span & 5.46 & $3-9$ & 1.45 \\
\hline Stockings of Cambridge & 6.49 & $3-10$ & 1.90 \\
\hline Intra/Extra Dimensional Set Shifting & 8.10 & $7-9$ & 0.93 \\
\hline Pattern Recognition Memory & 86.95 & $50-100$ & 9.49 \\
\hline \multicolumn{4}{|l|}{ Digit Span } \\
\hline Japanese & 11.52 & $4-22$ & 3.39 \\
\hline Portuguese & 9.88 & $3-15$ & 2.65 \\
\hline \multicolumn{4}{|l|}{ Language proficiency } \\
\hline \multicolumn{4}{|l|}{ Japanese } \\
\hline PVT-R score & 32.13 & $0-77$ & 9.79 \\
\hline PVT-R age & 6.60 & $3-12.3$ & 2.63 \\
\hline J. coss & 67.78 & $29-79$ & 12.8 \\
\hline \multicolumn{4}{|l|}{ Portuguese } \\
\hline TVf-usp & 91.54 & $28-131$ & 22.9 \\
\hline Test of Grammatical Comprehension of Portuguese Sentences ${ }^{b}$ & 17.21 & $2-20$ & 3.70 \\
\hline \multicolumn{4}{|l|}{ Number of years of parental education (years) } \\
\hline Mother & 11.23 & $8-15$ & 2.08 \\
\hline Father & 9.84 & $8-15$ & 1.78 \\
\hline
\end{tabular}

${ }^{a}$ Cognitive capacity was examined using CANTAB tasks. Digit span was examined using the Japanese and Portuguese versions of the WISC-III.

${ }^{\mathrm{b}}$ This test was created in Japan and was not used in Brazil.

CANTAB: Cambridge Neuropsychological Test Automated Battery;

PVT-R: Picture Vocabulary Test-Revised;

J.COSS: Japanese Test for Comprehension of Syntax and Semantics;

TVf-usp: Teste de Vocabulario auditivo por Figuras USP;

WISC-III: Wechsler Intelligence Scale for Students-Third Edition. SD: standard deviation 
Table 4 Explanatory variables affecting academic performance and behavioral problems in the Typical Group ( $\mathbf{N}=68)$

\begin{tabular}{|c|c|c|c|c|c|c|}
\hline \multirow[t]{2}{*}{ Variable } & \multicolumn{3}{|c|}{ Academic performance } & \multicolumn{3}{|c|}{ Behavioral problems } \\
\hline & Coef. & SE & $p$ & Coef. & SE & $p$ \\
\hline Paternal education & & & & -1.160 & 0.576 & 0.049 \\
\hline Digit span in Japanese & 0.181 & 0.059 & 0.004 & & & \\
\hline J.coss & & & & -0.287 & 0.088 & 0.002 \\
\hline
\end{tabular}

Japanese Test for Comprehension of Syntax and Semantics (J.COSS) were significantly associated with the Teacher's Report Form T-score. Estimates showed that scores for behavioral problems decreased by 1.160 points with an increase of 1 year in parental education, and decreased by 0.287 points with an increase of one point in J.COSS score. Other explanatory variables, such as place of birth, gender and Portuguese proficiency, were not found to be associated with either academic performance or behavioral difficulties.

\section{Discussion}

In this section, we return to the three research questions initially presented.

Are there Japanese-Brazilian students who are enrolled in mainstream classes, but are at risk of cognitive dysfunction?

The nonverbal cognition tasks revealed six students within the clinical range (At-risk Group). This means that $8 \%$ of participants had not been diagnosed with difficulties but still face risks because of their cognitive capacity. This is a slightly higher percentage than data reported by MEXT (2012), which suggests that $6.5 \%$ of children in mainstream classes in Japan have developmental disorders. Literature has indicated that CLD children are often underdiagnosed and remain in mainstream classes with few opportunities for appropriate support because the factors causing problems are not easily identified. Our data confirmed that such students also exist in the Japanese-Brazilian population.

Children in this group are considerably disadvantaged by the lack of support available to them. They may well fall behind with schoolwork or demonstrate inappropriate classroom manners. Children in our At-risk Group received poorer evaluation from classroom teachers in both academic and behavioral performance than children without risk. Their academic performance rating was even lower than that of children already diagnosed. Hibel and Jasper (2012) noted concern about disadvantages due to delays in intervention. Our results underscore the need for children in this group to be immediately, and thoroughly assessed so that a diagnosis can be given and appropriate support offered.

Are there Japanese-Brazilian students evaluated as having low academic achievement and behavioral difficulties in the absence of a recognized cognitive disorder?

Of participants with typical cognitive capacity, more than $70 \%$ received academic level ratings below grade level. This means that most of the CLD students in our sample 
have difficulties with their studies. As for the behavior rating, nearly $30 \%$ fall into the clinical/borderline range as having serious behavioral problems. Compared with data reported by Kawauchi et al. (2013), the proportion is higher than that of Japanese students, where $7.6 \%$ were in the clinical/borderline range. Our data confirmed that CLD students exhibit troubled behavior more frequently than monolingual peers, while they nevertheless have no cognitive delays. Leaving these children in classrooms without any intervention or support may cause further negative outcomes and a lack of educational success in their school careers (Kohler and Lazarín 2007; Tillman et al. 2006). If CLD students do not obtain appropriate support, they may drop out of school. Violence and significant rates of high school dropout have been reported by the same group of researchers. In order to reduce these children's problems and utilize their strengths, it is necessary to first recognize the affected students and then to identify the factors causing difficulties.

Are limited academic progress and behavioral difficulties influenced by three predictors: cognition, language and parental education?

\section{Factors affecting learning}

Recent studies have focused on working memory as one of the predictors of learning. Results of the present study, however, did not identify visuo-spatial working memory as a significant factor influencing learning evaluation. Instead, auditory working memory tested in the digit span task in Japanese was shown to be an influencing factor.

The question arises why the performance of the digit span task in Portuguese was not identified as a predictive factor. As rating was made based on students' classroom performance using Japanese as an official language, it is highly probable that the teacher's rating is influenced by a student's level of Japanese. However, it should be noted that proficiency in Japanese grammar and vocabulary did not influence learning evaluation. It could be that their receptive Japanese language skills, or nonverbal working memory in isolation, do not directly influence the teachers' evaluation in school subjects. However, once tasked with assignments with a greater processing load, they may show serious difficulties in thinking, working, and communicating using Japanese. Interaction between the two factors may cause CLD children's poor performance. Another possibility is that children's verbal productive skills generally influence teachers' evaluations. Since we did not collect data on language production, the question remains to be explored in future research.

Working memory capacity has been shown to be influential for learning. As Gathercole et al. (2008) indicated, teachers should focus on students whose working memory is restricted. These students need appropriate support with classroom activities so that the demands of processing auditory instructions and producing output are managed. The participants in this study demonstrated Japanese receptive vocabulary below grade level. With this in mind, Japanese-Brazilian students who are struggling academically should receive appropriate support in classroom activities and with their Japanese language development.

\section{Factors affecting behavioral problems}

The results of our multi-regression analysis revealed that Japanese grammatical skill and father's educational background have a major influence on the child's level of 
behavioral difficulties. We discuss why these two factors could affect the children's classroom behavior.

First, it should be noted that Japanese grammatical skill, not vocabulary size, emerged as a predictor of behavioral problems. While there is a considerable body of research addressing the association between language and behavioral problems (Lindsay et al. 2007; Pierce et al. 2013; Van Daal et al. 2007 few studies have considered grammatical understanding as a significant indicator. Petersen et al. (2013) argued that not only vocabulary size, but also other linguistic factors such as discourse and grammar, should be considered as influential factors to be targeted when improving behavioral problems. The effects of those other aspects of language have not yet been considered, but our data demonstrate that grammar development does influence student behavior.

One way to reduce CLD children's behavioral problems is through improving their Japanese syntactic skills. Our study revealed that children's average grammatical skills in Japanese were at the first grade level, while their mean chronological age was 9.4 years old. Improving their abilities to structure Japanese may lead to an improvement in classroom behavior. Although receptive vocabulary in Japanese was not a significant factor, the level was also found to be low, akin to that of a first grader (6.6-year-old). This suggests they need continuous support with other aspects of language, e.g., vocabulary, as well. While such support for CLD students may be helpful, it is also necessary for teachers to elaborate on their classroom instructions and to take learners limited language proficiency into account. Using uncomplicated grammar and vocabulary or visual aids to support their verbal message may be effective in assisting these children.

Another significant factor affecting student behavior is father's educational background. This finding does not coincide with past reports, showing greater influence of mother's educational background (Carneiro et al. 2013; Tamis-LeMonda et al. 2009). The difference may have arisen because other studies have focused primarily on monolingual children, and CLD families tend to be socially and economically vulnerable. As socioeconomic status has considerable impact on children (Bradley and Corwyn 2002), our CLD children who showed troubled behavior could also be affected by the socioeconomic status of the family. This study faced difficulties in asking parents for information about their income. We used parents' educational background as an indicator of the family's socioeconomic status, since there is generally a strong association with fathers' educational background and socioeconomic status (see Lamerz et al. (2005) who also use paternal education as an alternative indicator of economic status).

CLD children's home environment should be carefully considered by taking the social context of immigrants into account. Reports on Japanese-Brazilian populations in Japan note that many fathers are engaged in heavy manual labor and have relatively low levels of education (Liu et al. 2014) suggesting it may be difficult for them to be actively involved with their children's education. Research has found that parents' stressors are associated with poverty, and there is an emphasis on the importance of support and intervention for families (Ceballos and Bratton 2010; Sung 2014), which may play an important role in providing students with psychological stability and in improving their behavior.

\section{Conclusion}

The present study showed that there are Japanese-Brazilian students enrolled in mainstream classrooms who are at risk of cognitive dysfunction despite having never been 
diagnosed as having a developmental disorder. Such students urgently require appropriate support. Japanese-Brazilian students with typically developing cognitive capacity were also noted to show difficulties with their learning and classroom behavior, which teachers considered lower than the grade level. These two poor ratings of classroom performance were found to be individually associated with different factors.

The findings have important significance for educational activities in schools. CLD students are often suspected of having developmental disorders owing to the delay in classroom language acquisition, while there are students who should be referred for their developmental difficulties but are dismissed as struggling with the second language. The findings presented here may contribute to a better understanding of the causes underlying CLD students' classroom challenges. If these factors are elucidated, teachers may be able to develop their teaching methods or intervene in the home environment based on individual students' profiles.

As for the factors affecting academic achievement, Japanese working memory was found to be significant. Teachers should consider that struggling students may have limited verbal working memory capacity, and try to reduce the classroom processing load accordingly. Behavioral challenges were found to be affected by Japanese grammatical skill and home environment. Teachers should concern themselves more with supporting these children's syntactic skills and vocabulary development. They also need to engage more with students' home environments and try to enhance the parental communication.

Problems experienced by CLD students are caused by various factors. Appropriate support for these children should be considered carefully from the multiple perspectives of cognition, language and home environment. School staff have a key role to play in reducing difficulties of CLD students and improving their learning and behavior.

\section{Limitations}

In this study we were not able to collect data from Japanese monolingual students. The analysis focused on CLD students in the absence of a comparison group. The sample size was small and did not allow us to generalize the results obtained to the broader population of CLD students in Japan. We only assessed receptive language skills and cognitive function tasks administered were kept to a minimum. This is because we aimed to balance reasonably comprehensive data collection with awareness of children's limited capacity. A further limitation is that we identified children in the clinical range of developmental disorders, but could not provide further information about the nature of their difficulties or what their diagnosis may be. There are some tasks or questionnaires that are sensitive to specific developmental disorders but were not used here; e. g., the theory of mind task is sensitive to children with social and communication difficulties such as autism; The Children's Communication Checklist - Second Edition (Bishop and 2003) provides information about whether children have problems with specific language skills or social-pragmatic abilities. Finally, we could not directly collect data on children's socioeconomic status such as home income, since in the preliminary study parents and schools showed strong resistance to our request for the information. While the study was limited in these ways, it is significant that quantitative data in this area were obtained for the first time in Japan. 


\section{Methods}

\section{Participants}

Participants were recruited from public elementary schools in City A, located in the central area of Japan's main island. Foreigners make up 2.6\% of the population of City A, and there are 104 public elementary schools with 515 Japanese-Brazilian students. The Japanese-Brazilian students comprise $57.7 \%$ of the foreign students in these schools. The authors invited the principals of all 104 elementary schools to participate in the study after receiving approval from the Board of Education. A total of 14 principals expressed their willingness to participate. The 14 schools had 213 Japanese-Brazilian students, and of these, 81 were included in the study sample after their parents completed consent forms (participation rate: $38 \%$ ).

Of these 81 students, seven had previously been diagnosed with a disability (five with an intellectual disability enrolled in classes for special needs education, and one each with a developmental delay and ADHD enrolled in mainstream classes). These students were referred to as the Diagnostic Group.

The participants without diagnosis consisted of 20 students in grades 1 and 2 (27.2\%), 25 students in grades 3 and 4 (34.6\%) and 29 students in grades 5 and 6 (38.3\%). They were further divided into an At-risk and a Typical Group based on the scores obtained from the cognitive assessment task.

This study was approved by the Medical Ethics Review Committee of Kanazawa University on January 16, 2013 (number 1308) and adhered to the tenets of the Declaration of Helsinki.

\section{Measures}

We collected data on the children's cognitive capacity, language proficiency, and home environment as independent variables. Data were also obtained from teachers who were asked to rate the children's academic performance and behavioral problems as dependent variables.

\section{Cognitive capacity}

In order to reduce the influence of cultural background and language skills, we evaluated participants' nonverbal cognition. The Cambridge Neuropsychological Test Automated Battery system (CANTAB) was chosen since the tasks can mostly be administered nonverbally, reducing language and cultural influences (Roque et al. 2011). CANTAB was applicable to our participants since it contains normative data for children. Four tasks were selected. Three of these focused on testing executive functions, and one on visual memory, based on previous work by Roque et al. (2011). The tasks chosen were: Spatial Span (SSP); Stockings of Cambridge (SOC); Intra/Extra Dimensional Set Shifting (IED); and Pattern Recognition Memory (PRM). These tasks were conducted according to the test administration guide (Cambridge Cognition 2012).

In addition to the tests detailed, Digit Span tasks were conducted in Japanese and Portuguese. These were added to our study so that we could measure participants' verbal working memory, which could also influence children's classroom performance.

Spatial Span (SSP) tests participants' working memory capacity. In this task, several white boxes are shown in various locations on the screen. The color of the boxes 
changes into blue, one by one in a random order. Participants are required to remember the order of the change and asked to touch the box in the order they recall. The score ranges from 0 to 9 .

Stockings of Cambridge (SOC) assesses participants' spatial planning and motor control. Participants are shown two displays, each presenting three colored balls and containers. Aiming to make an identical pattern with the model shown on the upper display, participants are required to move the balls from one location to another under the restricted rules. The score ranges from 0 to 10.

Intra/Extra Dimensional Set Shifting (IED) aims to assess participants' rule acquisition and attentional set shifting. Participants are shown a pair of figures and asked to choose one of them, followed by feedback if it was right or wrong. They gradually learn the rule to obtain the right answer, but at some point the sorting rule changes without notice. Participants are expected to be aware of the change and flexibly follow the new rule. The score ranges from 0 to 12 .

Pattern Recognition Memory (PRM) tests participants' visual recognition memory. Participants are presented with a series of visual stimuli, one at a time, in the center of the screen and are asked to remember each of them. They are then presented with pairs of figures similar to each other and required to choose which of the two they have seen. The proportion correct is shown as the result (0-100).

Digit span task assesses verbal working memory capacity (Baddeley 2000). In this study, the forward and backward digit span tests were adopted from Japanese and Portuguese versions of the Wechsler Intelligence Scale for Children - Third Edition (WISC-III: Wechsler, 1998). In the forward digit span task, participants listen to a series of digits (e.g., "8-2-5") and repeat them immediately after the model is presented, while in the backward task, participants need to reverse the order of the numbers, starting from the last number to the first (e.g., "5-2-8"). Scores ranges from 0 to 32.

We aimed to identify students who are enrolled in mainstream classes but are at risk for cognitive difficulties. Luciana and Nelson (2002) used z-scores to divide subjects' performance into seven levels: Very Superior $(+3)$, Superior $(+2)$, High average $(+1)$, Average (0), Low Average $(-1)$, Borderline $(-2)$, Impaired ( -3$)$. They also showed means, standard deviations and ranges of the scores for each task according to age. Following the criteria used in this previous study, our participants with scores within the borderline/impaired range $(\mathrm{z}$-score $\leq-2)$ for at least one of the CANTAB tasks were categorized into the At-risk Group. Children who scored above the cutoff point were categorized into the Typical Group.

\section{Language proficiency}

In order to assess proficiency in both Japanese and Portuguese, four tests were used.

The Picture Vocabulary Test-Revised (PVT-R) is a standardized test, which derives participants' verbal mental age in Japanese. Participants are asked to select the picture corresponding to the word spoken by an examiner from an array of four pictures (Ueno et al. 2008). The score ranges from 0 to 89 , and the vocabulary age ranges from 3.0 to 12.25 years old.

The Japanese Test for Comprehension of Syntax and Semantics (J.COSS) was produced by Nakagawa et al. (2005) in accordance with the Test for Reception of 
Grammar (TROG) (Bishop 1989). J.COSS assesses grammatical skill in Japanese. Participants are required to choose the picture corresponding to the sentence given by an examiner from an array of four pictures. The score ranges from 0 to 80 .

The Teste de Vocabulario auditivo por Figuras USP (TVfusp) tests participants' vocabulary development in Portuguese. The examiner produces a word and the participant must choose the corresponding picture from an array of four pictures. The score ranges from 0 to 139 (Capovilla and Prudêncio 2006).

The Test of Grammatical Comprehension of Portuguese Sentences is an unpublished assessment developed by Japanese researchers, based loosely on the format of TROG. This test assesses grammatical skill in Portuguese. Participants are required to choose the picture corresponding to the sentence spoken by the examiner from an array of four pictures. The score ranges from 0 to 20 .

\section{Home environment}

Parents filled out a questionnaire regarding the students' place of birth and their own educational background. Other information such as the number of years of schooling was not included in the analysis, but was used as reference data.

Teacher ratings of academic performance and behavioral problems

Teacher's Report Form for Ages 6-18 (TRF/6-18) Japanese version contains questions regarding children's academic performance and their behavioral problems in class.

Academic performance was rated by their teachers on a five-point scale: far below grade level (1); somewhat below grade level (2); at grade level (3); somewhat above grade level (4); and far above grade level (5). Performance for the two core school subjects, mathematics and Japanese language, is rated in this questionnaire. The score ranges from 2 to 10 .

Behavioral problems were rated based on 118 items that describe students' behavioral problems such as "(the child acts) too young for his/her age" and "(the child has) difficulty in following directions". Teachers were asked to give one of the following scores to each question: Not True (as far as you know) (0): Somewhat or Sometimes True (1); and Very True or Often True (2). T-scores were derived from the results and used to evaluate the children's behavior. A cutoff point (60) was determined based on standardized data and scores falling above this level represented children with a clin$\mathrm{ical} /$ borderline level in behavior. T-scores ranged from 32 to 100.

\section{Procedures}

A bilingual Japanese-Brazilian collaborator whose native language is Portuguese explained the purpose of the study and the testing procedures to the parents at each school. She also administered TVfusp, the Test of Grammatical Comprehension of Portuguese Sentences, CANTAB tasks and the digit span task in Portuguese to our participant children. PVT-R, J.COSS and digit span in Japanese were administered by the first author. These tests were administered to each student in two sessions lasting approximately 90 minutes in total. The participants remained at school after class and were tested individually in a quiet room. Teachers and parents were given the relevant questionnaires to complete. 


\section{Statistical analysis}

The data for academic performance and behavioral problems were compared across the Typical, At-risk and Diagnostic Groups using the Wilcoxon rank-sum (MannWhitney) test. In order to explore the causes of poor academic achievement and behavioral problems, multiple regression analysis was conducted. The Diagnostic and At-risk Groups were excluded from this analysis since we aimed to ascertain whether students without any diagnosis or risk of cognitive disorder nevertheless showed difficulties in the classroom. Two dependent variables were established from the results of the TRF: academic performance and behavior. The explanatory variables are the scores of cognitive tasks (SSP, SOC, IED, PRM, Digit Span task in Japanese and Portuguese), the scores or ages obtained from language tests (PVT-R, J.COSS, TVfusp, Test of Grammatical Comprehension of Portuguese Sentences), and other factors regarding child or parental profiles (age, gender, place of birth, parental education). Using STATA/SE 13 software (Stata Corp., College Station, TX, USA), a $p$-value of less than 0.05 was considered significant in the stepwise multiple regression method.

\footnotetext{
Abbreviations

ADHD: Attention deficit hyperactivity disorder; CANTAB: Cambridge Neuropsychological Test Automated Battery; CLD: Culturally and Linguistically Diverse; ESL: English as a second language; IED: Intra/Extra Dimensional Set Shifting; J. COSS: Japanese Test for Comprehension of Syntax and Semantics; MEXT: Ministry of Education, Culture, Sports, Science and Technology-Japan; PRM: Pattern Recognition Memory; PVT-R: Picture Vocabulary Test-Revised; SD: Standard deviation; SOC: Stockings of Cambridge; SSP: Spatial Span; TRF: Teacher Rating Form; TROG: Test for Reception of Grammar; TVfusp: Teste de Vocabulario auditivo por Figuras USP; WISC-III: Wechsler Intelligence Scale for Students - Third Edition.
}

Competing interests

The authors declare that they have no competing interests.

\section{Authors' contributions}

YM and MO conceived of the study, and participated in its design and coordination and helped to draft the manuscript. All authors read and approved the final manuscript.

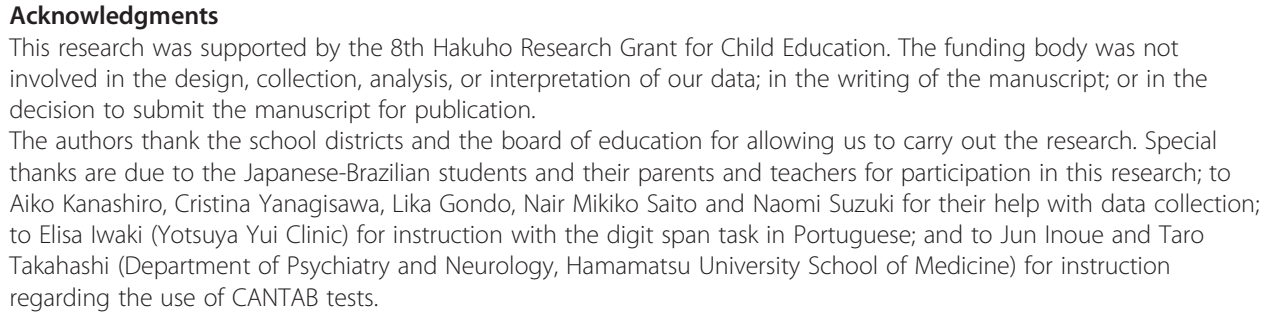

\section{Author details}

${ }^{1}$ United Graduate School of Child Development, Osaka University, Kanazawa University, Hamamatsu University School of Medicine, Chiba University and University of Fukui, Yamadaoka Suita, Osaka 565-0871, Japan. ${ }^{2}$ Research Center for Child Mental Development, Kanazawa University, Takaramachi, Kanazawa, Ishikawa 920-8640, Japan. ${ }^{3}$ United Graduate School of Child Development Osaka University, Kanazawa University Hamamatsu University School of Medicine, Chiba University and University of Fukui, Takaramachi, Kanazawa, Ishikawa 920-8640, Japan.

Received: 24 October 2014 Accepted: 19 March 2015

Published online: 10 April 2015

\section{References}

Abe, Yu. 2007. Zainichi gaikokujin jido seito no seishinteki shomondai to tabunkateki sien. Tokyo University of Foreign Studies Center for Multilingual Multicultural Education and Research Tagengo Tabunka kyodo jissen kenkyu 2: 102-110. Baddeley, Alan. 2000. The episodic buffer: a new component of working memory? Trends in Cognitive Sciences 4: 417-423. Bishop, Dorothy VM. 1989. Test for reception of grammar (TROG), Medical research council. London, UK. Bishop, Dorothy VM. 2003. The children's communication checklist. 2nd edition, Harcourt Assessment. London, UK. Bradley, Robert H, and Robert F Corwyn. 2002. Socioeconomic status and child development. Annual Review of Psychology 53(1): 371-399.

Bradley, Robert H, Robert F Corwyn, Margaret Burchinal, Harriette Pipes McAdoo, and Cynthia García Coll. 2001. The Home Environments of Children in the United States Part II: Relations with Behavioral Development through Age Thirteen. Blackwell: Publishers. 
Bradley, Renée, Louis Danielson, and Jennifer Doolittle. 2007. Responsiveness to Intervention: 1997 to 2007. Teaching Exceptional Children 39(5): 8-12.

Brydges, S, R Christopher, Corrine L Reid, Allison M Fox, and Anderson Mike. 2012. A unitary executive function predicts intelligence in children. Intelligence 40: 458-469.

Cambridge Cognition. 2012. CANTABeclipse 5 Test Administration Guide. Cambridge, UK: Cambridge Cognition Limited.

Capovilla, LF, Fernando César, and Érica R Prudêncio. 2006. Teste de vocabulário auditivo por figuras: normatização e validação preliminares. Avaliação psicológica 5(2): 189-203.

Carneiro, Pedro, Costas Meghir, and Matthias Parey. 2013. Maternal education, home environments, and the development of children and adolescents. Journal of the European Economic Association 11: 123-160

Carter-Smith, Karin. 2008. Response to Intervention (RTI). Great Neck Publishing.

Ceballos, Peggy L, and Sue C Bratton. 2010. Empowering Latino families: effects of a culturally responsive intervention for low-income immigrant Latino parents on children's behaviors and parental stress. Psychology in the schools 47(8): 761-775. doi:10.1002/pits.20502.

Corbett, Blythe A, Laura J Constantine, Robert Hendren, David Rocke, and Sally Ozonoff. 2009. Examining executive functioning in children with autism spectrum disorder, attention deficit hyperactivity disorder and typical development. Psychiatry Research 166: 210-222. doi:10.1016/j.psychres.2008.02.005.

Cummins, Jim. 1981. Age on arrival and immigrant second language learning in Canada: a reassessment. Applied Linguistics 2: 132-149.

Cummins, Jim. 2009. Transformative multiliteracies pedagogy: school-based strategies for closing the achievement Gap. Multiple Voices for Ethnically Diverse Exceptional Learners 11: 38-56.

Cummins, Jim. 2014. Beyond language: academic communication and student success. Linguistics and Education 26: 145-154. doi:10.1016/j.linged.2014.01.006.

Duncan, Greg J, Jeanne Brooks-Gunn, and Pamela Kato Klebanov. 1994. Economic Deprivation and Early Childhood Development, 296-318.

Dunn, Lloyd M. 1968. Special education for the mildly retarded: is much of it justifiable? Exceptional Children 35: 5-22.

Edwards, Nicole Megan. 2014. Number of children associated with mothers' perceived need for behavior support: Implications for parenting interventions. Journal of Child \& Family Studies 23: 527-536. doi:10.1007/s10826-013-9712-7.

Engel de Abreu Pascale, MJ, Martine Baldassi, Marina L Puglisi, and Debora M Befi-Lopes. 2013. Cross-linguistic and cross-cultural effects on verbal working memory and vocabulary: testing language-minority children with an immigrant background. Journal of Speech, Language \& Hearing Research 56: 630-642. doi:10.1044/1092-4388 (2012/12-0079)

Friedman, Naomi P, Akira Miyake, P Robin, Susan E Corley, John C De Fries, and John K Hewitt. 2006. Not all executive functions are related to intelligence. Psychological Science 17: 172-179. doi:10.1111/j.14679280.2006.01681.x.

Gathercole, Susan E, Emily Durling, Matthew Evans, Sarah Jeffcock, and Sarah Stone. 2008. Working memory abilities and children's performance in laboratory analogues of classroom activities. Applied Cognitive Psychology 22: 1019-1037. doi:10.1002/acp.1407.

Georgiades, Katholiki, Michael H Boyle, and Eric Duku. 2007. Contextual influences on children's mental health and school performance: the moderating effects of family immigrant status. Child Development 78(5): 1572-1591. doi:10.1111/j.1467-8624.2007.01084.x.

Golash-Boza, Tanya. 2005. Assessing the advantages of bilingualism for the children of immigrants. International Migration Review 39(3): 721-753.

Gunderson, Lee, and Linda S Siegel. 2001. The evils of the use of IQ tests to define learning disabilities in first- and second-language learners. Reading Teacher 55(1): 48-55.

Hammer, Carol Scheffner, Eugene Komaroff, L Barbara, Lisa M Rodriguez, Shelley E Scarpino, and Brian L Goldstein. 2012. Predicting Spanish-English bilingual children's language abilities. Journal of Speech, Language \& Hearing Research 55(5): 1251-1264. doi:10.1044/1092-4388(2012/11-0016).

Harry, Beth, and Janette K Klingner. 2014. Why are so many minority students in special education? Understanding race \& disability in schools, 2nd ed. New York: Teachers College Press.

Hibel, Jacob, and Andrea D Jasper. 2012. Delayed special education placement for learning disabilities among children of immigrants. Social Forces 91(2): 503-530. doi: 10.1093/sf/sos092.

Hughes, Charles A, and Douglas D Dexter. 2011. Response to intervention: a research-based summary. Theory Into Practice 50(1): 4-11. doi:10.1080/00405841.2011.534909.

Kawauchi, Mie, Nozomi Kihara, Yutaro Setoya, Hazuki Makino, Michiko Kita, and Yasuko Kanbayashi. 2013. Standardization of teacher's report form for ages 6-18 (TRF/6-18) Japanese version. Psychiatria et Neurologia Paediatrica Japonica 53: 211-223.

Kitawaki, Yasuyuki. 2008. Japan's migration policy: analysis of policy concepts and a study of National and Local Government Policies. Journal of multilingual multicultural studies and practices. 1: 5-25.

Klingner, Janette K, and Patricia A Edwards. 2006. Cultural considerations with response to intervention models. Reading Reseach Quaterly 41(1): 108-117. doi:10.1598/RRQ.41.1.6.

Kohler, Adriana D, and Melissa Lazarín. 2007. Hispanic education in the United States. National Council of La Raza, Statistical Brief 8: 1-16.

Lamerz, A, C Wehle, N Bruning, B Herpertz-Dahlmann, J Kuepper-Nybelen, H Brenner, G Trost-Brinkhues, and J Hebebrand. 2005. Social class, parental education, and obesity prevalence in a study of six-year-old children in Germany. International Journal of Obesity 29(4): 373-380. doi:10.1038/sj.jj.0802914.

Lezak, Muriel D. 1982. The problem of assessing executive functions. International Journal of Psychology 17(1-4): 281-297. doi:10.1080/00207598208247445.

Lindsay, Geoff, E Dockrell Julie, and Strand Steve. 2007. Longitudinal patterns of behaviour problems in children with specific speech and language difficulties: child and contextual factors. British Journal of Educational Psychology 77(4): 811-828. doi:10.1348/000709906X171127. 
Liu, Xiangying, Nakata Teruko, Yoshida Yukie, Kuriyama Yoko, and Chen Hueichen. 2014. An investigative study into the life and child-nurturing environment of foreign workers' families in Japan: mainly the Japanese Brazilian families who are living in Aichi Prefecture, 39-49.

Luciana, Monica. 2003. Practitioner review: computerized assessment of neuropsychological function in children: clinical and research applications of the Cambridge Neuropsychological Testing Automated Battery (CANTAB). Journal of Child Psychology \& Psychiatry \& Allied Disciplines 44(5): 649-663.

Luciana, Monica, and A Charles Nelson. 2002. Assessment of neuropsychological function through use of the Cambridge neuropsychological testing automated battery: performance in 4- to 12-year-old children. Developmental Neuropsychology 22(3): 595-624.

McFadden, Karen E, Catherine S Tamis-LeMonda, and Natasha J Cabrera. 2011. Quality matters: low-income fathers' engagement in learning activities in early childhood predict children's academic performance in fifth grade. Family Science 2(2): 120-130. doi: 10.1080/19424620.2011.655952.

Ministry of Education, Culture, Sports, Science and Technology - Japan. 2012a. Koritsu gakko ni zaiseki shiteiru gaikokujinjidoseitosu. Gakko Kihon Chosa.

Ministry of Education, Culture, Sports, Science and Technology - Japan. International Education Division. 2012b. "Nihongosido ga hitsuyo na jidoseito no ukeire jokyo ni kansuru chosa" no kekka ni tsuite.

Miyake, Akira, and Naomi P Friedman. 2012. The nature and organization of individual differences in executive functions: Four general conclusions. Current Directions in Psychological Science 21(1): 8-14. doi:10.1177/0963721411429458.

Nakagawa, Yoshiko, Koyama Takamasa, and Suga Tetsuo. 2005. Development of receptive grammar in Japanese children: an investigation using the J.COSS. The Japanese Journal of Developmental Psychology 16(2): 145-155.

Nii, Kimiko, and Yururi Makoto. 2013. New framework of educational assessment for foreign children: based on results from a survey of out of school children in Hamamatsu. Journal of the Research on Lifelong Learning and Career Education 9: 1-12.

Norbury, Courtenay Frazier, and Sparks Alison. 2013. Difference or disorder? Cultural issues in understanding neurodevelopmental disorders. Developmental Psychology 49(1): 45.

Pennington, Bruce F, and Sally Ozonoff. 1996. Executive functions and developmental psychopathology. Journal of Child Psychology and Psychiatry 37(1): 51-87.

Petersen, Isaac T, John E Bates, Brian M D'Onofrio, Claire A Coyne, Jennifer E Lansford, Kenneth A Dodge, Gregory S Pettit, and Carol A Van Hulle. 2013. Language ability predicts the development of behavior problems in children. Journal of Abnormal Psychology 122(2): 542-557.

Pierce, Margaret E, Adrianna Wechsler-Zimring, Gil Noam, Maryanne Wolf, and Tami Katzir. 2013. Behavioral problems and reading difficulties among language minority and monolingual urban elementary school students. Reading Psychology 34(2): 182-205. doi:10.1080/02702711.2011.626108.

Pina, Violeta, Luis J Fuentes, Alejandro Castillo, and Sofia Diamantopoulou. 2014. Disentangling the effects of working memory, language, parental education and non-verbal intelligence on children's mathematical abilities. Frontiers in Psychology 5. doi: 10.3389/fpsyg.2014.00415.

Reynolds, Cecil R, and Sally E Shaywitz. 2009. Response to intervention: prevention and remediation, perhaps. Diagnosis, No. Child Development Perspectives 3(1): 44-47. doi:10.1111/j.1750-8606.2008.00075.x.

Roque, Daniela Tsubota, Rosani Aparecida Antunes Teixeira, Elaine C Zachi, and Dora F Ventura. 2011. The use of the Cambridge neuropsychological test automated battery (CANTAB) in neuropsychological assessment: application in Brazilian research with control children and adults with neurological disorders. Psychology \& Neuroscience 4(2): 255-265. doi:10.3922/j.psns.2011.2.011.

St Clair-Thompson, Helen L, and Susan E Gathercole. 2006. Executive functions and achievements in school: shifting, updating, inhibition, and working memory. The Quarterly Journal of Experimental Psychology 59(4): 745-759. doi:10.1080/17470210500162854.

Suárez-Orozco, Carola, Allyson Pimentel, and Margary Martin. 2009. The significance of relationships: Academic engagement and achievement among newcomer immigrant youth. The Teachers College Record 111(3): 712-749.

Sullivan, Amanda L. 2011. Disproportionality in special education identification and placement of English language learners. Exceptional Children 77(3): 317-334.

Sung, Youngji. 2014. Differential effect of social-emotional behaviors on academic achievement of language-minority students. Child Youth Care Forum 43: 393-416. doi:10.1007/s10566-014-9245-2.

Tamis-LeMonda, Catherine S, Rahil D Briggs, Sandra G McClowry, and David L Snow. 2009. Maternal control and sensitivity, child gender, and maternal education in relation to children's behavioral outcomes in African American families. Journal of Applied Developmental Psychology 30(3): 321-331. doi:10.1016/j.appdev.2008.12.018.

Tillman, Kathryn Harker, Guang Guo, and Kathleen Mullan Harris. 2006. Grade retention among immigrant children. Social Science Research 35: 129-156. doi:10.1016/j.ssresearch.2004.07.001.

Ueno, Kazuhiko, Naoko Nagoshi, and Satoru Konuki. 2008. PVT-R manual. Tokyo: Nihon Bunka Kagakusha.

Van Daal, John, Ludo Verhoeven, and Hans Van Balkom. 2007. Behaviour problems in children with language impairment. Journal of Child Psychology \& Psychiatry 48(11): 1139-1147. doi:10.1111/j.1469-7610.2007.01790.

Villiger, Caroline, Christian Wandeler, and Alois Niggli. 2014. Explaining differences in reading motivation between immigrant and native students: The role of parental involvement. International Journal of Educational Research 64: 12-25. doi:10.1016/j.jijer.2013.10.004.

Vuontela, Virve, Synnöve Carlson, Anna-Maria Troberg, Tuija Fontell, Petteri Simola, Suvi Saarinen, and Eeva T Aronen. 2013. Working memory, attention, inhibition, and their relation to adaptive functioning and behavioral/emotional symptoms in school-aged children. Child Psychiatry \& Human Development 44: 105-122. doi:10.1007/s10578-012-0313-2.

Wechsler, D. 1998. Wechsler intelligence scales for Students, 3rd ed. San Antonio: The Psychological Corporation. 